\title{
LEVANTE DE CARPA COMÚN (Cyprinus carpio) EN SISTEMAS CERRADOS DE RECIRCULACIÓN, BAJO CONDICIONES DE LABORATORIO
}

Fecha de recepción: 11 de noviembre de 2015 • Fecha de Evaluación: 6 de mayo de 2016 • Fecha de aceptación: 10 de junio de 2016 - Disponible en línea: 25 de julio de 2016

\section{REARING OF COMMON CARP (Cyprinus carpio) IN RECIRCULATING AQUACULTURE SYSTEM UNDER LABORATORY CONDITIONS}

Jesús Carrascal R1, B.Sc., Ana Torres-Mesa1', B.Sc., Hernán Hurtado-Giraldo1, Ph.D., Edwin Gómez-Ramírez,2, M.Sc.

\section{RESUMEN}

La acuicultura es una industria de gran crecimiento en busca de sistemas más amigables con el ambiente. La producción de carpa común mundialmente se presenta principalmente en China, lo que ha permitido que sea la especie de mayor producción. Por lo tanto evaluar sistemas donde el recurso del agua sea mejor aprovechado es de gran importancia. Se evaluó el crecimiento y parámetros productivos de Cyprinus carpio en sistemas cerrados de recirculación (SCR). Para esto se realizaron dos experimentos (M1 y M2) con 5 SCR donde se mantuvieron los peces por un periodo de 12 semanas de cultivo cada uno. En cada SCR se cultivaron 100 alevinos. Las variables evaluadas fueron peso, longitud estándar y longitud total, y se calcularon parámetros productivos. Se hizo el seguimiento y control de los parámetros físico-químicos del agua. De acuerdo a lo observado los peces de M1 alcanzaron un peso promedio de $6.13 \pm 0.80 \mathrm{~g}$, longitud estándar $5.66 \pm 0.24 \mathrm{~cm}$ y longitud total $7.08 \pm 0.23 \mathrm{~cm}$ mientras en M2 alcanzaron un peso de $10.22 \pm 0.89 \mathrm{~g}$, longitud estándar $6.62 \pm 0.18 \mathrm{~cm}$ y longitud total $8.2 \pm 0.22 \mathrm{~cm}$. El mejor crecimiento en M2 se relacionó con valores de parámetros fisicoquímicos dentro del rango adecuado para la especie. El crecimiento en peso y en longitud se ajustó al modelo exponencial en M1 y M2. En conclusión puede decirse que el cultivo inicial de alevinos de carpa en SCR permite buenos crecimientos y supervivencia empleando poca agua y bajo condiciones altamente controladas.

Palabras clave: Sistemas de recirculación, crecimiento, calidad de agua, iniciación, desarrollo sostenible.

1 Universidad Militar Nueva Granada, Facultad de Ciencias Básicas y Aplicadas, Programa de Biología Aplicada, Grupo de Ictiología, Cajicá (Cundinamarca), Colombia

2 Correspondencia: edwin.gomez@unimilitar.edu.co 


\section{ABSTRACT}

Aquaculture is a major growth industry that is looking of more environmentally friendly production systems. Common carp production worldwide is mainly in China, which has allowed it to be the kind of major crop. Therefore evaluate systems where water is better utilized it is of great importance. The evaluation of $C$. carpius growth, survival and productive parameters in recirculating aquaculture system (RAS) was made. It takes 12 weeks for carried out two culture experiments. The experiment used 5 RAS each one with 100 fry. Body weight, standard length and total length were evaluated, and productive parameters were calculated. Control and supervision of physicochemical parameters were made. M1 fishes attained mean body weigth of $6.13 \pm 0.80$ $\mathrm{g}$, standard length of $5.66 \pm 0.24 \mathrm{~cm}$ and total length of $7.08 \pm 0.23 \mathrm{~cm}$, while in M2 these figures were $10.22 \pm$ $0.89 \mathrm{~g}, 6.62 \pm 0.18 \mathrm{~cm}$ and $8.2 \pm 0.22 \mathrm{~cm}$, respectively. Better growth in M2 was related with physicochemical parameters level within the range reported for this species. Weight and length growth did adjust to an exponential model in M1 and M2. In conclusion, initiation of common carp fry in RAS of low technology, did allows for good growth and survival, with low water use, and under highly controled conditions.

Key words: recirculating aquaculture systems, growth, water quality, sustainable development.

\section{INTRODUCCIÓN}

Cyprinus carpio pertenece a la familia de los $\mathrm{Ci}$ prínidos. Es una especie de hábitos omnívoros y sus requerimientos alimenticios no son exigentes (Horvath et al. 2002). Tolera una amplia variedad de condiciones ambientales como temperatura, $\mathrm{pH}$ y dureza del agua lo que ha permitido su manejo en diversos sistemas de producción acuícola (Yilmaz et al. 2005). El incremento en la demanda de productos del sector acuícola ha generado la necesidad de aumentar la eficiencia del cultivo de esto organismos (Casas, 2008).

En Colombia comúnmente se utilizan sistemas de producción extensivos o semi-intensivos que toleran densidades no mayores a $1-3 \mathrm{pez} / \mathrm{m}^{2}$ (Salazar, 2001), lo cual no podrá cubrir la demanda de productos del sector acuícola que ha generado la necesidad de aumentar la eficiencia del cultivo de estos organismos (Casas, 2008). Para aumentar la densidad de cultivo, se debe enfrentar factores limitantes como bajos niveles de oxígeno disuelto (OD) y acumulación de desechos tóxicos (Casas, 2008). Para dar solución a dichos problemas, en la acuicultura se utilizan los sistemas cerrados de recirculación (SRC) (Timmons y Ebeling, 2007). En estos sistemas se suministra OD y se controla la calidad del agua. Los SCR han obtenido buenos resultados en el aumento de las densidades de producción de peces, con disminución del consumo de agua, aumento en la eficiencia en el uso del espacio, bajo impacto ambiental y generación constante de producto de buena calidad (Yousef et al. 2003; Timmons y Ebeling, 2007). El objetivo del estudio fue evaluar el inicio de C. carpio en SCR, determinando la cinética de crecimiento y supervivencia, y llevando un control de las condiciones físico-químicas del agua con el fin de determinar el desempeño de estos sistemas, y posible uso para la etapa inicial de cultivo. 


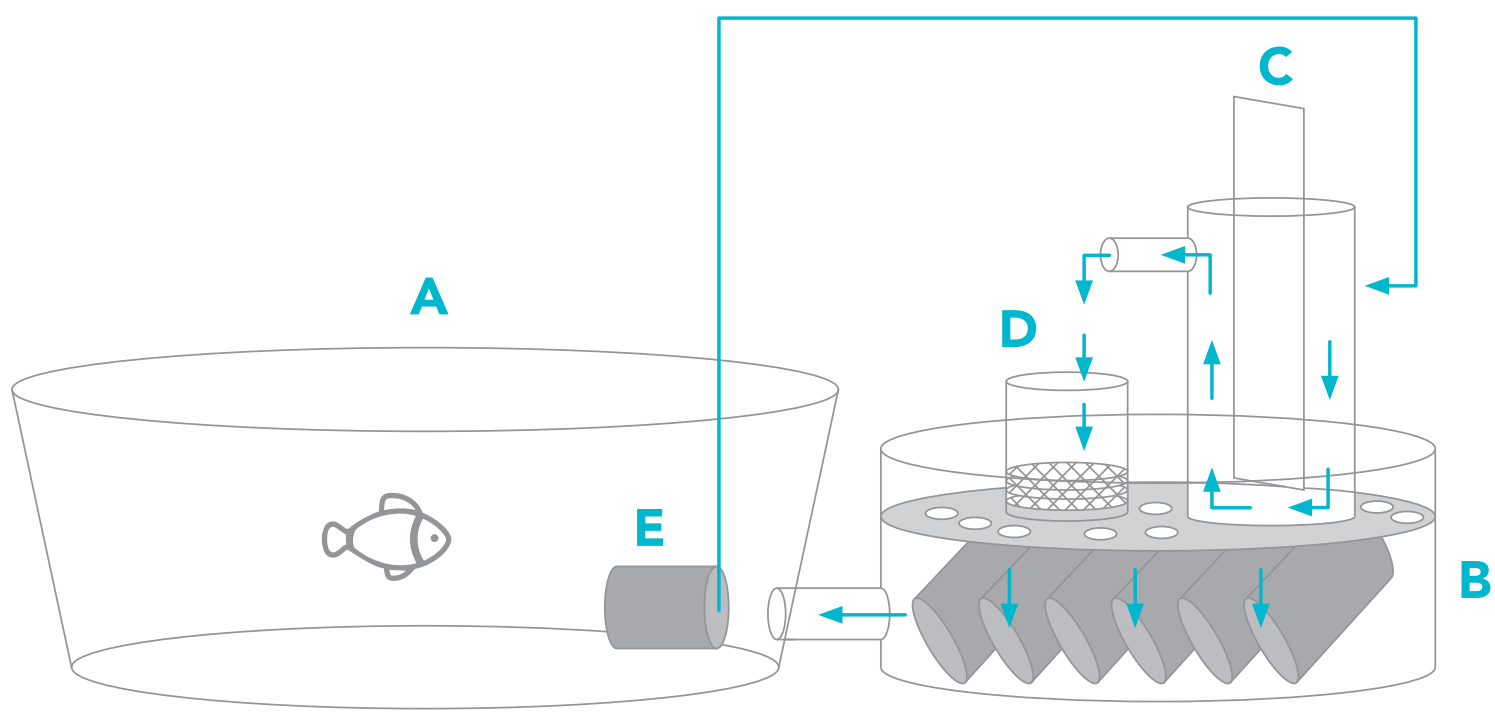

\section{MATERIALES Y MÉTODOS}

\section{Lugar de experimentación}

Este trabajo se llevó a cabo en el laboratorio de Fisiología Animal de la Facultad de Ciencias Básicas y Aplicadas, Programa de Biología Aplicada, Universidad Militar Nueva Granada, en el municipio de Cajicá (Cundinamarca) a 2558 msnm, y temperatura promedio ambiente de $14^{\circ} \mathrm{C}$.

\section{Montaje de sistemas de recirculación (SCR)}

Cada SCR estaba compuesto por un tanque de 250 L para mantenimiento de los peces, el cual tenía en su interior un aireador de $6 \mathrm{~L} / \mathrm{min}$, dos termostatos de 100 W y una bomba sumergible de $1500 \mathrm{~L} / \mathrm{h}$ que transportaba el agua al clarificador (Fig. 1). Este último era un tanque plástico de $15 \mathrm{~L}$ con una lámina de poliestireno de $1.5 \mathrm{~cm}$ de espesor que funcionaba como barrera física para disminuir la velocidad del flujo de agua, permitiendo precipitar residuos sólidos en suspensión. Luego el agua circulaba por gravedad a un filtro mecánico, construido con un tanque

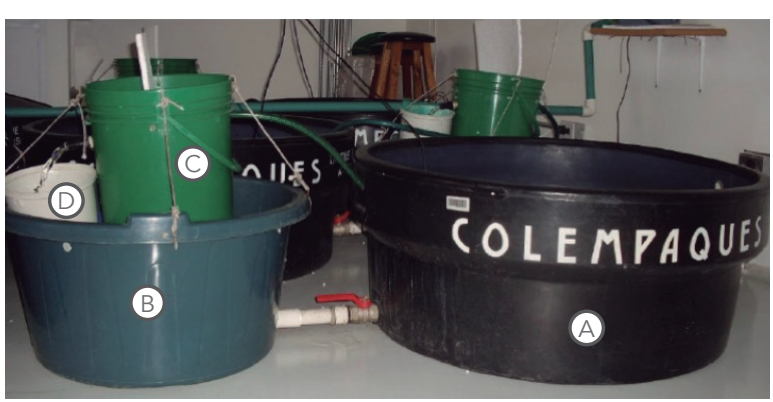

Figura 1. Prototipo de SCR. A: tanque de peces, B: biofiltro, C: clarificador, D: filtro mecánico, E: bomba de agua. Las flechas verdes indican la dirección del agua a través del sistema.

plástico de $3 \mathrm{~L}$, con seis discos de malla de anjeo con un ojo de $1 \mathrm{~mm}$ de diámetro, los cuales se cortaron de acuerdo al diámetro del tanque. El agua filtrada pasaba al biofiltro por gravedad. El biofiltro estaba constituido por un tanque plástico de $60 \mathrm{~L}$, dentro del cual se encontraban 6 paquetes de anjeo de $50 \mathrm{~cm} \times 25$ $\mathrm{cm}$ con esferas de poliestireno, que servían de sustrato para las bacterias nitrificantes, proporcionando un 
área de aproximadamente $90000 \mathrm{~cm}^{2}$, es importante señalar que el biofiltro presentaba aireación constante. Finalmente, una tubería de 1" conectaba al biofiltro con el tanque de peces (Fig. 1 y 2).

Antes de iniciar los experimentos los sistemas fueron inoculados con bacterias de otros sistemas maduros para lo cual se dispuso de paquetes de anjeo con esferas de poliestireno y se pasó alrededor de $50 \mathrm{~L}$ agua. Después se colocaron 6 individuos de Carassius auratus adultos por sistema para acelerar la maduración y mantener las bacterias inoculadas previamente (Timmons y Ebeling, 2007).

\section{Adquisición de los peces}

Los alevinos fueron adquiridos en una empresa comercial de peces de consumo. Los alevinos se mantuvieron en cuarentena 24 días dentro de otro SCR, antes de pasar a las unidades experimentales.

\section{Diseño experimental}

Para evaluar el funcionamiento de los SCR se realizaron dos experimentos de levante de C. carpio en dos momentos o tiempos diferentes, los cuales se denominaron $\mathrm{M} 1$ y $\mathrm{M} 2$, cada uno con 5 repeticiones y con una duración de 12 semanas de cultivo (84 días). Transcurrido este tiempo se procedía a adquirir una nueva cohorte de peces y se repetía el estudio de levante por otras 12 semanas. En cada SCR se sembraron 100 alevinos de C. carpio, con un
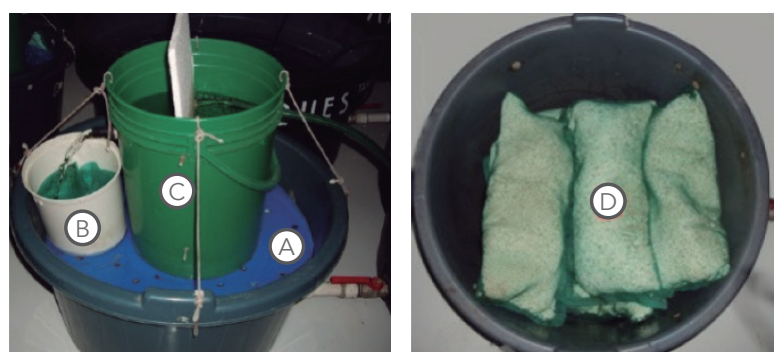

Figura 2. Detalle del prototipo del SCR. A: biofiltro, B: filtro mecánico, C: clarificador, y D: componentes del biofiltro, paquetes de anjeo con las bolas de poliestireno. peso inicial de $1.40 \pm 0.05 \mathrm{~g} /$ ind para M1 y $1.33 \pm$ $0.03 \mathrm{~g} /$ ind para M2.

\section{Alimentación}

Los peces se alimentaron 4 veces al día (8:00 h, $12: 00$ h, 15:00 h y 17:00 h) con alimento comercial Truchina ${ }^{\circledR}$ al $45 \%$ de proteína cruda, ajustada al 10\% del peso total promedio de los peces muestreados de cada sistema hasta que alcanzaron un peso promedio de $3 \mathrm{~g}$, luego se alimentaron al 5\% con la misma frecuencia. La cantidad de alimento y el porcentaje de alimentación se ajustaron con base a los datos de crecimiento tomados cada semana de muestreo.

\section{Variables de crecimiento}

Cada 12 días se realizaron muestreos de 30 individuos escogidos al azar de cada uno de los SCR tanto para M1 como para M2. A estos se les tomaron medidas de peso, por medio de una balanza analítica $\left(\mathrm{OHAUS}{ }^{\circledR}\right.$, Ohaus Corporation, Parsippany, NJ, USA), longitud total (LT) y longitud estándar (LS), con ayuda de un ictiómetro. Se calcularon los siguientes parámetros productivos: Incremento de peso (IP) $=$ (peso final-peso inicial); ganancia en peso (GP) = ((peso final-peso inicial)/peso inicial)*100; tasa de crecimiento absoluto (TCA) = (peso final-peso inicial)/(tiempo final-tiempo inicial); factor de condición $K=$ (peso final/longitud estándar final $\left.{ }^{3}\right)^{\star} 100$; factor de conversión alimenticia $($ FCA) $=$ total alimento consumido/(biomasa final-biomasa inicial); tasa de eficiencia alimentaria (TEA) = (biomasa final-biomasa inicial)/total alimento consumido; tasa de eficiencia protéica $(T E P)=$ (biomasa final-biomasa inicial)/proteína consumida y supervivencia $(S)$ = (número inicial de peces/número final de peces) ${ }^{\star} 100$

\section{Medición de parámetros fisicoquímicos}

Semanalmente en cada uno de los SCR tanto en $\mathrm{M} 1$ y $\mathrm{M} 2$, se realizó un registró de los siguientes 
parámetros; nitrógeno amoniacal total (NAT), nitrito, nitrato, oxígeno disuelto (OD), temperatura y dureza general (GH), no obstante, para el pH y la dureza de carbonatos $(\mathrm{KH})$ en $\mathrm{M} 1$ se midió una vez a la semana, y en $\mathrm{M} 2$ tres veces por semana y se realizaban correctivos de calidad de agua. Para ello, se utilizaron test TETRA ${ }^{\circledR}$ (TETRA, Melle, Alemania), un potenciómetro (SCHOTT ${ }^{\circledR}$, Schott Instruments, Mainz, Alemania) y para la temperatura se utilizó un termómetro de máximas y mínimas.

La temperatura en todos los SCR en M1 y M2 se mantuvo entre 22 y $24^{\circ} \mathrm{C}$ y el OD entre $4-5 \mathrm{mg} / \mathrm{L}$. El principal reactivo usado para corregir la calidad de agua fue la adicción de bicarbonato de sodio, el cual fue el $25 \%$ del peso del alimento suministrado al día, esto con el fín de proveer carbono a las bacterias nitrificantes y mejorar la capacidad buffer (Timmons y Ebeling, 2007). Además, semanalmente se hacía reposición del agua perdida por evaporación, mantenimiento y limpieza de sólidos a los filtros mecánicos, clarificadores, biofiltros, bombas y tubería. Diariamente se hicieron observaciones de presencia o ausencia de síntomas de enfermedades y de los cambios en el comportamiento de alimentación y nado de los peces.

\section{Análisis estadístico}

El análisis estadístico se realizó con el programa $R$ de libre distribución en internet. Se utilizó un diseño completamente aleatorio, expresando los resultados obtenidos como promedio ( \pm desviación estándar). A los datos se les hizo una prueba de Shapiro-Wilk para determinar si las variables respuesta presentaban una distribución normal. Adicionalmente se realizó una prueba T para determinar si había diferencia entre M1 y M2. Se realizaron curvas de crecimiento de las carpas, utilizando como parámetros la longitud estándar, longitud total, y el peso corporal (Zar, 2009). Se definió a partir de un análisis de regresión, si el crecimiento se ajustaba más a un modelo exponencial o a uno lineal.

\section{RESULTADOS Y DISCUSIÓN}

Para el crecimiento de $C$. carpio se obtuvieron diferencias significativas $(p<0.05)$ entre el crecimiento de los peces de M1 y M2 (Tabla 1). En un periodo de 84 días, los peces del M1 alcanzaron un peso promedio de 6,13 $\pm 2,00 \mathrm{~g}$ con una longitud total de $7,1 \pm 0,8 \mathrm{~cm}$; y los peces del M2 alcanzaron un peso promedio de 10,22 $\pm 3,39 \mathrm{~g}$ con una longitud total de $8,2 \pm 0,8 \mathrm{~cm}$. Esta diferencia $(p<0.05)$ también es observada en el ajuste de las variables de crecimiento de M1 y M2 a los modelos evaluados y en los parámetros productivos de estos dos montajes.

Tabla 1. Promedio inicial y final de las variables de crecimiento de C. carpio en SCRA para cada uno de

Tabla 1.

\begin{tabular}{|c|c|c|c|c|c|c|c|c|}
\hline \multirow{2}{*}{ 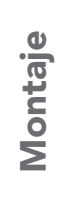 } & \multicolumn{2}{|c|}{ Peso (g) } & \multicolumn{2}{|c|}{ LE (cm) } & \multicolumn{2}{|c|}{ LT $(\mathrm{cm})$} & \multicolumn{2}{|c|}{ Biomasa (kg) } \\
\hline & $\frac{\bar{d}}{\frac{\mathbb{U}}{\mathrm{E}}}$ & 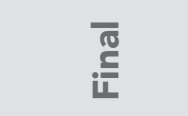 & $\frac{\bar{\sigma}}{\frac{\underline{U}}{\underline{E}}}$ & $\stackrel{\bar{\pi}}{\stackrel{\bar{x}}{i}}$ & 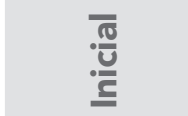 & $\stackrel{\bar{\pi}}{\stackrel{\bar{x}}{4}}$ & $\frac{\bar{\pi}}{\frac{\pi}{\underline{U}}}$ & $\begin{array}{l}\bar{T} \\
i \frac{1}{1}\end{array}$ \\
\hline M1 & $1.40 \pm 0.05 a$ & $6.13 \pm 0.80 a$ & $3.42 \pm 0.11 \mathrm{a}$ & $5.66 \pm 0.24 a$ & $4.34 \pm 0.05 a$ & $7.08 \pm 0.23 a$ & $0.14 \pm 0.004 a$ & $0.61 \pm 0.08 a$ \\
\hline M2 & $1.34 \pm 0.04 a$ & $10.22 \pm 0.89 b$ & $3.58 \pm 0.07 a$ & $6.62 \pm 0.18 b$ & $4.34 \pm 0.05 a$ & $8.2 \pm 0.22 b$ & $0.13 \pm 0.003 a$ & $1.02 \pm 0.09 b$ \\
\hline
\end{tabular}

ISSN 1900-4699 • Volumen 12 • Número 2 • Páginas 124-135 • 2016• DOI: http://dx.doi.org/10.18359/rfcb.2023 

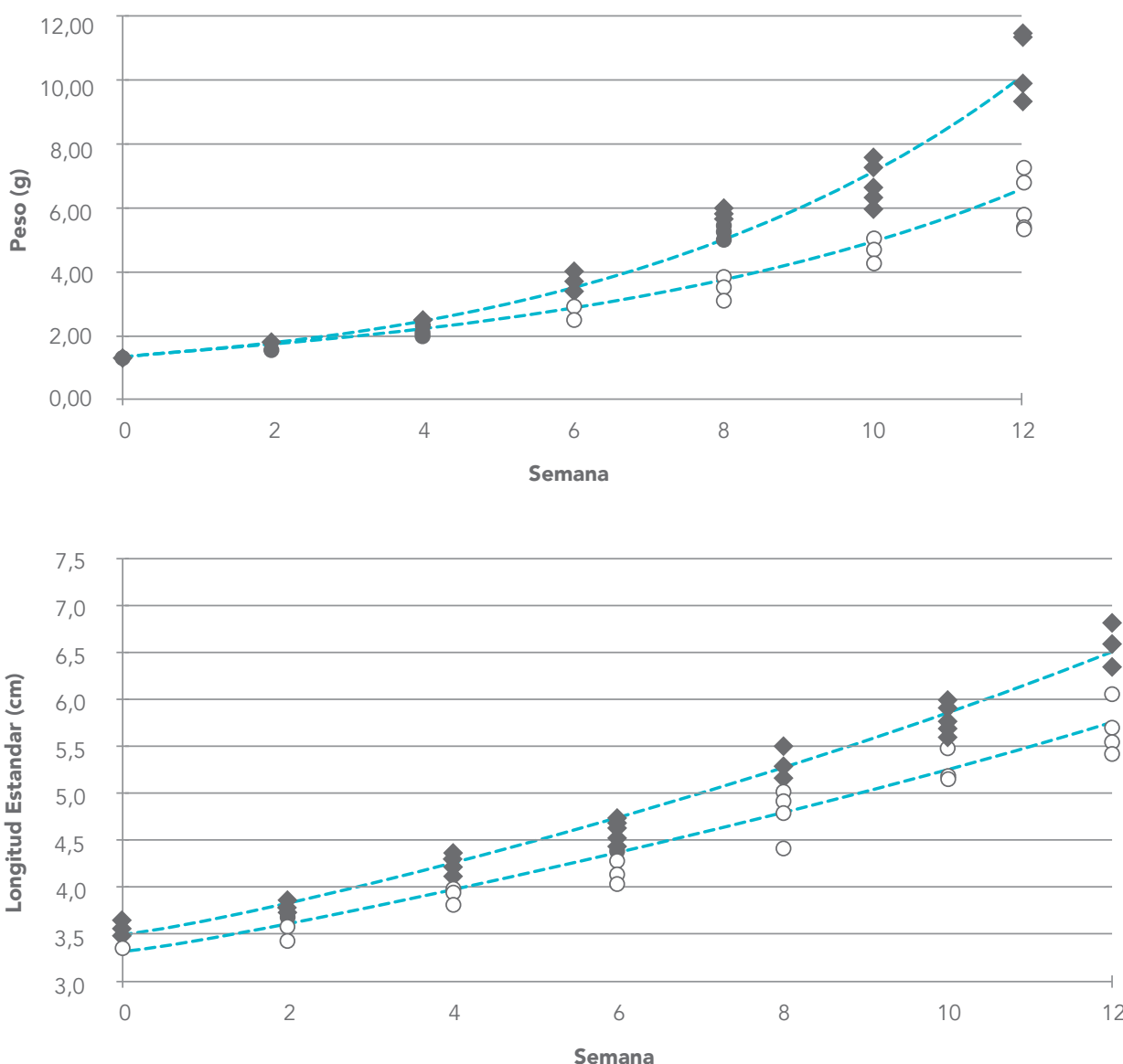

Semana

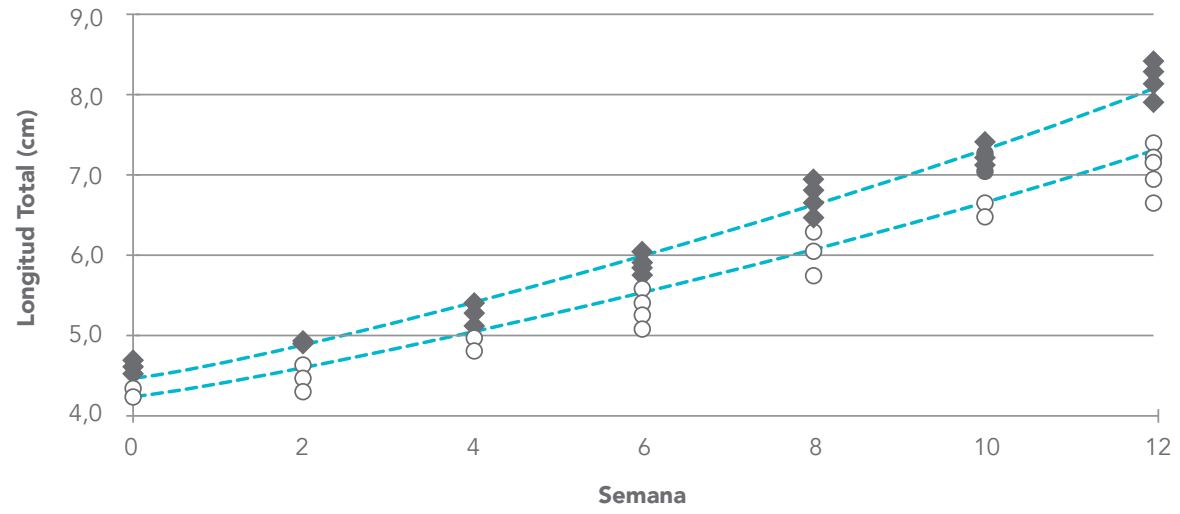

LT M1

LT M2

Figura 2. Crecimiento de C. carpio en SCRA. A. Peso. B. Longitud estándar. C. Longitud total. El trazo punteado representa la línea de tendencia exponencial. 
los tanques de M1 y M2. LE: longitud estándar, LT: longitud total. Comparación entre M1 y M2: valores con letras iguales no difieren significativamente $(p>0.05)$.

Los resultados arrojados por la evaluación de lo modelo potencial (Tabla 2) indican que el crecimiento de C. carpio en SCR a lo largo del estudio para $\mathrm{M} 1$ y $\mathrm{M} 2$ es de tipo isométrico, lo que revela que las variables evaluadas (peso y longitud) aumentan en igual proporción (Ricker, 1971). En M1 y M2 el peso de los peces se ajustó de mejor manera al modelo exponencial, sin embargo el coeficiente de determinación $\mathrm{R}^{2}$ indica que $\mathrm{M} 2$ se ajustó mejor en comparación a M1 (Tabla 2. Fig 3). La longitud total y estándar se ajustaron de manera similar al modelo exponencial y lineal, sin embargo hay una tendencia a ajustar mejor al modelo exponencial especialmente en M2. Esta dinámica de crecimiento para ambos experimentos concuerda con lo descrito para la mayoría de organismos biológicos que durante sus primeras etapas de desarrollo presentan un crecimiento exponencial (Ramírez, 1999).

En los parámetros productivos los peces de M2 mostraron en GP casi el doble de lo obtenido en M1, con base en esto la mayoría de los demás parámetros productivos de M2 fueron significativamente ( $p$ $<0.05$ ) superiores a lo conseguido en M1 (Tabla 3), esto indica que las condiciones de crecimiento en M2 permitieron un mejor crecimiento de los individuos. La S para ambos montajes fue alta. El factor de condición K, indicador numérico de la condición de los peces (grado de bienestar, robustez), que permite determinar las condiciones en que se logran los mejores rendimientos (Hernández et al. 2008) y la $S$ no mostraron diferencias significativas ( $p>0.05$ ). A pesar de la gran cantidad de estudios que se han

Tabla 2. Modelos de crecimiento evaluados en base a tres variables de crecimiento de C. carpio cultivados en SCR. $R^{2}$ : coeficiente de determinación, LE: longitud estándar, LT: longitud total, M: montaje.

\begin{tabular}{|c|c|c|c|c|c|c|c|}
\hline \multirow{2}{*}{ Montaje } & \multirow{2}{*}{ Variable } & \multicolumn{2}{|c|}{ M. Exponencial } & \multicolumn{2}{|r|}{ M. Lineal } & \multicolumn{2}{|c|}{ M. Potencial } \\
\hline & & $\mathbf{R}^{2}$ & Modelo & $\mathbf{R}^{2}$ & Modelo & $\mathbf{R}^{2}$ & Modelo \\
\hline \multirow{3}{*}{1} & Peso & 0.94 & $Y=1.3029 e^{0.1339 x}$ & 0.88 & $Y=0.4259 x+0.8227$ & \multirow[b]{2}{*}{0.99} & \multirow[b]{2}{*}{$Y=0.0192 x^{2.9325}$} \\
\hline & LT & 0.94 & $Y=4.221 e^{0.0453 x}$ & 0.93 & $Y=0.2545 x+4.1099$ & & \\
\hline & LE & 0.94 & $Y=3.3304 e^{0.0458 x}$ & 0.92 & $Y=0.2043 x+3.237$ & - & - \\
\hline \multirow{3}{*}{2} & Peso & 0.99 & $Y=1.2934 e^{0.1709 x}$ & 0.90 & $Y=0.7088 x+0.2747$ & \multirow[b]{2}{*}{0.99} & \multirow[b]{2}{*}{$Y=0.0082 x^{3.4013}$} \\
\hline & $\mathbf{L T}$ & 0.98 & $Y=4.4365 e^{0.05 x}$ & 0.97 & $Y=0.3065 x+4.2737$ & & \\
\hline & LE & 0.98 & $Y=3.4847 e^{0.052 x}$ & 0.96 & $Y=0.2539 x+3.3451$ & - & - \\
\hline
\end{tabular}


Tabla 3. Parámetros productivos del crecimiento de C. carpio en SCR para cada uno de los tanques para M1 y M2. Promedio, \pm desviación estándar de los parámetros productivos. Valores con letras iguales no difieren significativamente $(p>0.05)$.

\begin{tabular}{|c|c|c|c|c|c|c|c|c|}
\hline Montaje & IP (g) & GP (\%) & $\begin{array}{l}\text { TCA } \\
\text { (g/día) }\end{array}$ & $\mathbf{K}$ & FCA & TEA & TEP & S (\%) \\
\hline M1 & $\begin{array}{l}\sigma \\
\infty \\
\infty \\
0 \\
+1 \\
\stackrel{1}{0} \\
\stackrel{-}{\sigma}\end{array}$ & $\begin{array}{l}\sigma \\
\tilde{\sigma} \\
\tilde{D} \\
+1 \\
\stackrel{1}{\alpha} \\
\infty \\
m \\
m\end{array}$ & 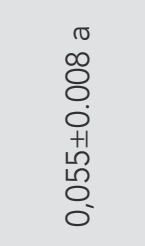 & $\begin{array}{l}\sigma \\
0 \\
\check{0} \\
\dot{0} \\
+1 \\
\stackrel{1}{n} \\
m \\
m\end{array}$ & $\begin{array}{l}\sigma \\
\text { N̦ } \\
0 \\
+1 \\
0 \\
0 \\
0\end{array}$ & $\begin{array}{l}\sigma \\
0 \\
0 \\
0 \\
+1 \\
\text { D } \\
\text { m } \\
0\end{array}$ & 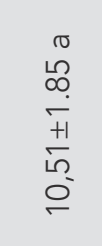 & 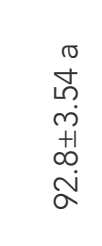 \\
\hline M2 & $\begin{array}{l}0 \\
\text { L } \\
\infty \\
\infty \\
+1 \\
+1 \\
\infty \\
\infty \\
\infty \\
\infty\end{array}$ & 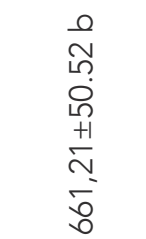 & $\begin{array}{l}0 \\
\stackrel{0}{0} \\
\vdots \\
0 \\
+1 \\
0 \\
0 \\
0\end{array}$ & $\begin{array}{l}\sigma \\
0 \\
0 \\
0 \\
0 \\
+1 \\
0 \\
n \\
n\end{array}$ & $\begin{array}{l}0 \\
0 \\
0 \\
0 \\
+1 \\
\hat{\alpha} \\
\vdots\end{array}$ & $\begin{array}{l}0 \\
\stackrel{0}{0} \\
0 \\
+1 \\
+1 \\
\tilde{+} \\
0\end{array}$ & $\begin{array}{l}\frac{0}{2} \\
\alpha \\
\frac{1}{+1} \\
\stackrel{1}{2} \\
\stackrel{-}{\sigma}\end{array}$ & $\begin{array}{l}\stackrel{\sigma}{\sim} \\
\underset{m}{+1} \\
+ \\
\infty \\
\sim \\
\sim\end{array}$ \\
\hline
\end{tabular}

hecho en C. carpio, no se encontró bibliografía con cual comparar los resultados de los parámetros productivos obtenidos en este trabajo.

En la tabla 4 se comparan los resultados del crecimiento de C. carpio obtenidos con algunos cultivos en estanque. Para esta comparación se tuvo como supuesto que los estanques de cultivo tenían una $1 \mathrm{~m}$ de profundidad (Peteri et al. 1992a; Peteri et al. 1992b; Mojica y Villaneda, 2001; CIFA, 2010). En cuanto a la tasa de crecimiento absoluto (TCA), para CIFA (2010) y los SCR (M2) se obtuvieron datos similares utilizando solamente el 9\% (L/ind) del agua de un cultivo en estanque y con 3 veces más individuos por metro cuadrado. En Peteri et al. (1992a) se observa claramente que en un tiempo similar y bajo las mismas condiciones de cultivo, a medida que la densidad es mayor, el crecimiento de los individuos disminuye significativamente. Esto es debido básicamente a que, a mayor densidad, mayor es la competencia por recursos, entre ellos, la comida. En los cultivos intensivos además de haber una gran competencia por comida también existe una competencia por el consumo de oxígeno disuelto, no solo entre peces, sino también con las bacterias que utilizan oxígeno para descomponer la materia orgánica (Timmons y Ebeling, 2007). Sin embargo, en las densidades de 12.5 y $14.0 \mathrm{ind} / \mathrm{m}^{2}$ el crecimiento de las carpas (Peteri et al, 1992a; Peteri et al. 1992b) en términos de TCA es comparativamente superior al obtenido en los SCR. Una de las posibles razones, corresponde a la práctica de fertilización de los estanques lo que promueve el crecimiento de materia orgánica y de microinvertebrados, incrementando el acceso al alimento por la generación de dos fuentes de alimento, como lo son, los concentrados, diseñados con base en los requerimientos alimenticios de los peces (observar las dietas utilizadas en Peteri et al. 1992a; Peteri et al. 1992b), y la otra fuente de alimento es el plancton (Peteri et al. 1992a; Peteri et al. 1992b). Es decir que si la densidad de siembra es comparativamente baja, la competencia por recursos también lo es. En los SCR las carpas no tenían 
acceso a plancton y no se conoce si el alimento suministrado a C. carpio cumplía con todos los requerimientos nutricionales para la especie.

Como además se observa en la tabla 5 el crecimiento de los peces de M1 y M2 presentaron diferencias, generado posiblemente por los parámetros de calidad de agua. En las primeras semanas, las cinco repeticiones de M1, presentaron valores fuera del rango de tolerancia para el pH, TAN y nitrito (Tabla 5). Los bajos niveles de $\mathrm{pH}$, posiblemente causados por la formación de ácido carbónico (Masser et al. 1999), pueden causar estrés, susceptibilidad a contraer enfermedades y disminución de crecimiento (PIR, 1999). Cuando existen altas concentraciones de nitrito éste puede entrar al torrente sanguíneo en cantidades tóxicas, oxidando el hierro en la molécula de hemoglobina de un estado ferroso a un estado férrico, resultando en un producto llamado metahemoglobina. Esta molécula posee poca afinidad con el oxígeno lo que resulta en una baja capacidad de transporte por todo el organismo (Jensen 2003; Kroupova et al. 2005).

El amonio no ionizado $\left(\mathrm{NH}_{3}\right)$ es un gas altamente soluble y posee una alta capacidad de difusión en soluciones acuosas lo que le permite viajar fácilmente a través de las células por medio de canales acuosos (Evans, 1998). Gracias a esta capacidad de difusión, se facilita la interacción con otras moléculas interfiriendo, como ocurre al combinarse con protones y elevar el $\mathrm{pH}$ intracelular y de la sangre a niveles incompatibles con funciones macromoleculares (Evans, 1998). El amonio ionizado $\left(\mathrm{NH}_{4}^{+}\right)$(que es menos soluble que el $\mathrm{NH}_{3}$ ) se mueve básicamente dependiendo de gradientes de concentración. La proporción del $\mathrm{NH}_{3}$ y $\mathrm{NH}_{4}{ }^{+}$en el agua depende del $\mathrm{pH}$ y la temperatura, de manera que cuando aumenta el $\mathrm{pH}$ y la temperatura, la forma tóxica del NAT tiende a incrementar su concentración (Hutchinson et al. 2004). En la tabla 5 se evidencian altas concentraciones de NAT, sin embargo los bajos niveles

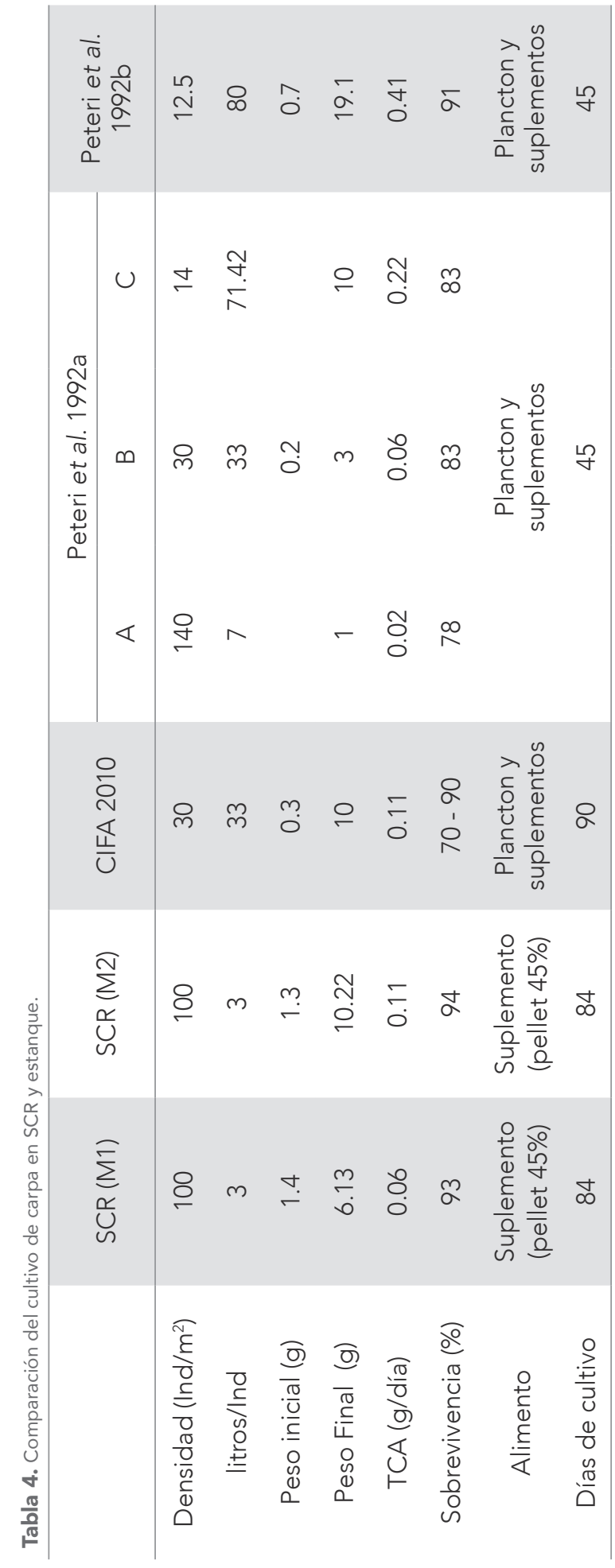




\begin{tabular}{|c|c|c|c|c|c|c|c|c|c|c|c|c|c|c|c|}
\hline & $\frac{I}{Q}$ & 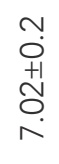 & 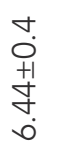 & 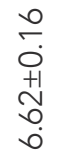 & $\begin{array}{l}\sim \\
0 \\
+1 \\
\infty \\
\sim \\
0\end{array}$ & $\begin{array}{l}\text { No } \\
\text { +1 } \\
\frac{1}{0}\end{array}$ & 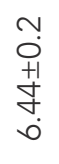 & $\begin{array}{l}N \\
0 \\
+1 \\
0 \\
0 \\
0\end{array}$ & $\begin{array}{l}\stackrel{\infty}{\check{0}} \\
+1 \\
+ \\
\text { m. } \\
0\end{array}$ & $\begin{array}{l}n \\
0 \\
+1 \\
0 \\
0 \\
0\end{array}$ & $\begin{array}{l}+ \\
\dot{0} \\
+1 \\
\text { m. } \\
0\end{array}$ & \begin{tabular}{l}
$\check{0}$ \\
+ \\
+1 \\
\multirow{2}{+}{} \\
0
\end{tabular} & $\begin{array}{l}a \\
\check{0} \\
+1 \\
0 \\
0 \\
0\end{array}$ & そ & ' \\
\hline & $\frac{I}{\underline{I}}$ & 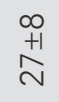 & 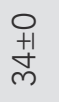 & 唄 & 足 & 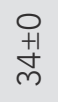 & $\begin{array}{l}\text { O } \\
\text { +1 } \\
\text { m }\end{array}$ & $\stackrel{\stackrel{ }{+1}}{\stackrel{2}{=}}$ & $\begin{array}{l}0 \\
+1 \\
\stackrel{1}{N}\end{array}$ & 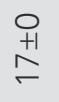 & 足 & 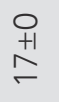 & 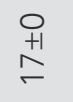 & そ & ' \\
\hline & $\underset{\mathbb{I}}{\mathrm{I}}$ & $\begin{array}{l}\stackrel{+}{+1} \\
\underset{\sim}{N}\end{array}$ & $\begin{array}{l}\stackrel{0}{+1} \\
\stackrel{N}{\sim}\end{array}$ & $\begin{array}{l}\stackrel{+}{+} \\
+1 \\
\stackrel{+}{E} \\
\rightleftharpoons\end{array}$ & $\begin{array}{l}\text { I } \\
+1 \\
0 \\
+ \\
+\end{array}$ & $\begin{array}{l}\stackrel{\sim}{+1} \\
\stackrel{+1}{m} \\
\stackrel{m}{\sim}\end{array}$ & 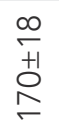 & $\begin{array}{l}\stackrel{\infty}{+1} \\
\stackrel{+}{\rightleftharpoons}\end{array}$ & $\begin{array}{l}\underset{+}{+1} \\
\underset{m}{\stackrel{P}{Ð}}\end{array}$ & $\begin{array}{l}\stackrel{0}{+1} \\
\stackrel{+1}{O} \\
\stackrel{0}{-}\end{array}$ & $\begin{array}{l}\frac{0}{+1} \\
\stackrel{+}{\rightleftharpoons} \\
\rightleftharpoons\end{array}$ & $\begin{array}{l}\frac{O}{+1} \\
\stackrel{+1}{\sigma} \\
=\end{array}$ & $\begin{array}{l}\stackrel{m}{+} \\
\stackrel{m}{\sim} \\
\stackrel{n}{\sim}\end{array}$ & $\begin{array}{l}\underset{N}{+} \\
+1 \\
\stackrel{1}{O} \\
\sim\end{array}$ & , \\
\hline & $\stackrel{\dot{m}}{\mathrm{O}}$ & 号 & 之 & そ & $\begin{array}{l}0 \\
\text { 임 } \\
8\end{array}$ & 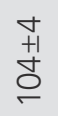 & 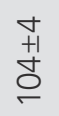 & 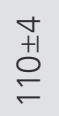 & 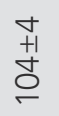 & $\begin{array}{l}\text { O } \\
+1 \\
\text { O } \\
\qquad\end{array}$ & 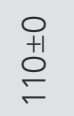 & $\begin{array}{l}0 \\
+1 \\
0 \\
0\end{array}$ & $\begin{array}{l}\text { ㅇ } \\
+1 \\
\text { O } \\
\leftarrow\end{array}$ & $\begin{array}{l}\underset{+}{+} \\
+1 \\
\infty \\
\stackrel{D}{ \pm}\end{array}$ & , \\
\hline & $\frac{\tilde{O}}{Z}$ & $\begin{array}{l}0 \\
+1 \\
\text { m } \\
0\end{array}$ & 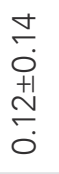 & $\begin{array}{l}+ \\
0 \\
+ \\
\text { + } \\
0 \\
0\end{array}$ & $\begin{array}{l}\text { on } \\
+1 \\
\stackrel{+}{0}\end{array}$ & $\begin{array}{l}0 \\
+1 \\
m \\
0\end{array}$ & $\begin{array}{l}0 \\
+1 \\
m \\
0\end{array}$ & 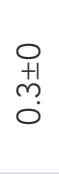 & $\begin{array}{l}0 \\
+1 \\
m \\
0\end{array}$ & $\begin{array}{l}0 \\
+1 \\
\text { m } \\
0\end{array}$ & $\begin{array}{l}0 \\
+1 \\
m \\
0 \\
0\end{array}$ & $\begin{array}{l}0 \\
+1 \\
m \\
0\end{array}$ & $\begin{array}{l}\check{0} \\
\text { +1 } \\
\stackrel{1}{0} \\
0\end{array}$ & $\begin{array}{l}0 \\
+1 \\
m \\
0\end{array}$ & ' \\
\hline$\stackrel{\mathfrak{N}}{\Sigma}$ & 安 & $\begin{array}{l}0 \\
+1 \\
0\end{array}$ & $\begin{array}{l}\text { பे } \\
0 \\
+1 \\
+1 \\
0 \\
0\end{array}$ & $\begin{array}{l}\circ \\
0 \\
+1 \\
0\end{array}$ & $\begin{array}{l}0 \\
\stackrel{0}{0} \\
+1 \\
\\
0\end{array}$ & $\begin{array}{l}0 \\
0 \\
+1 \\
0\end{array}$ & $\begin{array}{l}\circ \\
\circ \\
0 \\
0\end{array}$ & $\begin{array}{l}\circ \\
0 \\
+1 \\
0\end{array}$ & $\begin{array}{l}\circ \\
0 \\
+1 \\
0\end{array}$ & $\begin{array}{l}\circ \\
0 \\
+1 \\
0\end{array}$ & $\begin{array}{l}0 \\
0 \\
+1 \\
0\end{array}$ & $\begin{array}{l}\circ \\
0 \\
+1 \\
0\end{array}$ & 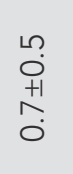 & 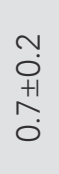 & ' \\
\hline & $\frac{I}{Q}$ & 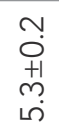 & $\begin{array}{l}\check{o} \\
+1 \\
\stackrel{+}{n}\end{array}$ & $\begin{array}{l}+ \\
0 \\
+ \\
\stackrel{+}{1} \\
\text { மn }\end{array}$ & $\begin{array}{l}m \\
0 \\
+1 \\
o \\
o \\
\dot{\sigma}\end{array}$ & $\begin{array}{l}m \\
0 \\
0 \\
+1 \\
0 \\
\text { in }\end{array}$ & 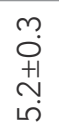 & 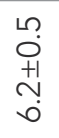 & $\begin{array}{l}n \\
0 \\
+1 \\
0 \\
0 \\
\text { in }\end{array}$ & $\begin{array}{l}0 \\
\text { O } \\
\stackrel{+}{N} \\
\text { o }\end{array}$ & 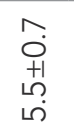 & $\begin{array}{l}\check{o} \\
+1 \\
\\
\end{array}$ & $\begin{array}{l}\bar{o} \\
\stackrel{+}{+1} \\
\overline{0}\end{array}$ & $\begin{array}{l}\hat{0} \\
+ \\
\stackrel{+}{+}\end{array}$ & $\begin{array}{l}\text { Ln } \\
\text { o } \\
\text { ஸे } \\
0\end{array}$ \\
\hline & $\frac{I}{\underline{y}}$ & $\stackrel{+}{\stackrel{+}{\rightleftharpoons}}$ & $\stackrel{\stackrel{ }{+1}}{\stackrel{2}{\rightleftharpoons}}$ & 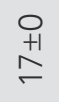 & $\stackrel{\stackrel{+}{+1}}{\stackrel{2}{L}}$ & $\stackrel{+}{\stackrel{+}{\perp}} \stackrel{-}{\leftarrow}$ & 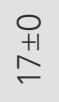 & $\begin{array}{l}\infty \\
+1 \\
\stackrel{1}{N}\end{array}$ & $\begin{array}{l}\text { O } \\
\text { +1 } \\
\text { m }\end{array}$ & $\begin{array}{l}\infty \\
+1 \\
+ \\
q\end{array}$ & 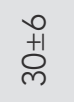 & $\begin{array}{l}0 \\
\stackrel{+}{+1} \\
m\end{array}$ & $\begin{array}{l}0 \\
+1 \\
\text { +1 } \\
m\end{array}$ & $\begin{array}{l}0 \\
+1 \\
0 \\
0\end{array}$ & $\frac{8}{\wedge}$ \\
\hline & $\frac{I}{\mathbb{N}}$ & i+ & $\frac{m}{\stackrel{+1}{n}}$ & $\frac{+}{+1}$ & 古 & 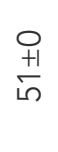 & $\frac{\stackrel{N}{+1}}{\stackrel{N}{n}}$ & $\frac{\stackrel{N}{+1}}{\stackrel{N}{r}}$ & $\underset{+\frac{m}{+1}}{\stackrel{m}{\sim}}$ & $\underset{+\underset{N}{N}}{\stackrel{+}{N}}$ & $\begin{array}{l}\stackrel{\text { N }}{+} \\
+1 \\
\stackrel{2}{\alpha}\end{array}$ & $\begin{array}{l}\stackrel{\curvearrowright}{+} \\
+1 \\
\stackrel{+}{\sigma}\end{array}$ & 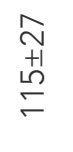 & $\begin{array}{l}\stackrel{\text { I }}{+} \\
\stackrel{ \pm}{ \pm}\end{array}$ & 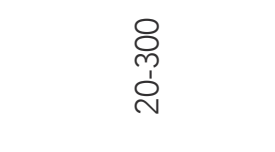 \\
\hline & @े & 吕 & 只 & $\frac{m}{\frac{m}{+1}}$ & $\frac{a}{++1}$ & $\frac{\stackrel{\sim}{+1}}{\stackrel{+}{+}}$ & 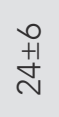 & $\begin{array}{l}\stackrel{0}{+1} \\
\stackrel{+1}{\sim} \\
\stackrel{f}{1}\end{array}$ & $\frac{\sigma}{\underset{+1}{+}}$ & $\frac{N}{+1}$ & $\frac{0}{+1}$ & 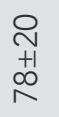 & $\begin{array}{l}\text { D } \\
\text { +1 } \\
\text { లn }\end{array}$ & そ & ষ্ণ \\
\hline & Oें & ○ & $\begin{array}{l}\stackrel{\circ}{+} \\
\stackrel{+1}{\check{r}}\end{array}$ & $\begin{array}{l}\text { ON } \\
+1 \\
+1 \\
0 \\
0\end{array}$ & $\begin{array}{l}0 \\
0 \\
+1 \\
m \\
m \\
0\end{array}$ & $\begin{array}{l}0 \\
0 \\
+1 \\
\text { m. } \\
0\end{array}$ & $\begin{array}{l}0 \\
0 \\
+1 \\
m \\
m \\
0\end{array}$ & $\begin{array}{l}0 \\
0 \\
+1 \\
m \\
m \\
0\end{array}$ & $\begin{array}{l}0 \\
0 \\
+1 \\
m \\
0 \\
0\end{array}$ & $\begin{array}{l}\bar{o} \\
+1 \\
\text { m. } \\
0\end{array}$ & $\begin{array}{l}0 \\
0 \\
+1 \\
m \\
m \\
0\end{array}$ & $\begin{array}{l}\bar{o} \\
+1 \\
\text { गे } \\
\text { o. }\end{array}$ & そ & そ & $\frac{8}{\dot{v}}$ \\
\hline 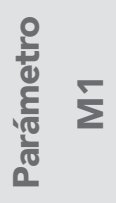 & 安 & $\begin{array}{l}\tilde{N} \\
0 \\
+1 \\
+ \\
0\end{array}$ & 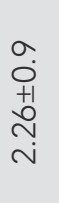 & $\begin{array}{l}\stackrel{\sim}{\sim} \\
\stackrel{+}{\sim} \\
\sim \\
m\end{array}$ & $\begin{array}{l}0 \\
0 \\
+1 \\
0 \\
0 \\
0\end{array}$ & $\begin{array}{l}\infty \\
+ \\
+1 \\
0 \\
0 \\
m \\
m\end{array}$ & $\begin{array}{l}\stackrel{0}{+} \\
+1 \\
\stackrel{+}{0} \\
\stackrel{+}{+}\end{array}$ & $\begin{array}{l}N \\
\stackrel{1}{+1} \\
\stackrel{+1}{0} \\
0\end{array}$ & 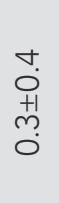 & $\begin{array}{l}0 \\
0 \\
+1 \\
m \\
m \\
0\end{array}$ & $\begin{array}{l}- \\
+1 \\
+1 \\
0 \\
0 \\
0\end{array}$ & $\begin{array}{l}\dot{+} \\
\text { o } \\
+1 \\
\dot{0} \\
\dot{0}\end{array}$ & そ & $\begin{array}{l}\stackrel{\sim}{\sim} \\
\stackrel{+}{+1} \\
\stackrel{+}{\leftarrow}\end{array}$ & $\frac{8}{i}$ \\
\hline$\stackrel{\varepsilon}{\Phi}$ & & 0 & $r$ & N & $m$ & $\forall$ & in & 0 & $\boldsymbol{N}$ & $\infty$ & $a$ & 움 & $\check{r}$ & $\stackrel{\sim}{\sim}$ & $\begin{array}{c}\text { Timmons } \\
\text { y Ebeling, } 2007\end{array}$ \\
\hline
\end{tabular}


de $\mathrm{pH}$ permitieron que el $\mathrm{NH}_{3}$ no sobrepasara los $0.008 \mathrm{mg} / \mathrm{l}$ en el peor de los casos, pero el NAT sí sobrepasó los niveles de tolerancia que es $2.0 \mathrm{mg} / \mathrm{l}$ (Timmons y Ebeling, 2007). Las concentraciones altas de NAT y nitrito posiblemente fueron producto de la falta de maduración del biofiltro. Esto se evidencia en el aumento de la concentración de nitrito y en las bajas concentraciones de nitrato durante las primeras cinco semanas (Tabla 5). Después de la semana 6, el NAT y el nitrito se mantuvieron generalmente en concentraciones dentro del rango de tolerancia (Timmons y Ebeling, 2007) y el nitrato mostró una tendencia a aumentar su concentración (Tabla 5).

Para los parámetros (Tabla 5) de pH y KH en M1, se observó que los niveles de $\mathrm{pH}$ y la concentración de carbonatos descendían rápidamente. Con base a la experiencia adquirida con el montaje $\mathrm{M} 1$, se hizo necesario un mejor seguimiento de estas variables para M2, con el fin de evitar que el pH estuviera fuera del rango de tolerancia de $C$. carpio y al mismo tiempo aumentar la capacidad buffer del agua y proporcionar carbonatos para los procesos de nitrificación bacteriana (Hutchinson et al. 2004; Timmons y Ebeling, 2007). En M2 el pH, el NAT, nitrito y nitrato se mantuvieron dentro del rango adecuado para $C$ carpio, (Tabla 7, 8 y 9). Sin embargo los niveles de carbonato no estuvieron dentro de los rangos descritos para el funcionamiento de los sistemas (Timmons y Ebeling, 2007), aun así con alcalinidad baja las bacterias realizaron el proceso de nitrificación de manera satisfactoria lo cual se evidenció en la transformación de NAT a nitrito y de nitrito a nitrato (Tabla 5).

\section{AGRADECIMIENTOS}

A la Vicerrectoría de Investigaciones de la Universidad Militar Nueva Granada (proyecto CIAS 1457), y a Colciencias (programa jóvenes investigadores).

\section{REFERENCIAS}

1. Casas D. 2008. Sistema de recirculación de agua para la cría intensiva de cachama blanca (Piaractus brachypomus). Trabajo de Grado. Programa de agronomía. Facultad de Agronomía. Universidad Centroccidental. Caracas, $97 \mathrm{p}$.

2. CIFA. 2010. Central Institute of Freshwater Aquaculture, Bhubaneshwar, Orissa. http:// www.cifa.in/Default.aspx. Accedida el 13 de octubre de 2013.

3. Evans D. 1998. The physiology of fishes. Second edition. CRC press LLC. 519 pp.

4. Horvath L, Tamas G y Seagrave C. 2002. Carp and pond fish culture. $2 d$ edition. Oxford, Blackwell Ltda, 185 p.

5. Hutchinson W, Mathew J, O'Sullivan D, Casemen D y Clarke S. 2004. Recirculating Aquaculture Systems: Minimum standards for design. Inland Aquaculture Association of South Australia, Kent, $70 \mathrm{p}$.

6. Jensen B. 2003. Nitrite disrupts multiple physiological functions in aquatic animals. Comparative Biochemical Physiology, 135:9-24.

7. Kroupova H, Machova J y Svobodova Z. 2005. Nitrite influence on fish: a review. Veterinary Medica Czech, 50:461-471. 
8. Masser M, Rakocy J y Losordo T. 1999. Recirculating aquaculture tank production systems. Management of Recirculating Systems. Southem Regional Aquaculture Center. United States Department of Agriculture. 9 pp.

9. Mojica H y Villaneda A. 2001. Construcción de estanques. En: Rodriguez H, Daza P, Carrillo M. (Eds.). Fundamentos de Acuicultura Continental. Bogotá, Instituto Nacional de Pesca y Acuicultura, $243 \mathrm{p}$.

10. Peteri A, Nandi S y Chowdowdhury S. 1992a. Manual on seed production of carps. Institutional Strengthening in the Fisheries Sector. Bangladesh. 61 pp. http://www.fao.org/docrep/field/003/ac376e/AC376E02.htm. Accedida el 13 de octubre de 2013.

11. Peteri A, Nandi S y Chowdowdhury S. 1992b. Development of large size carp fingerling production technology. Institutional Strengthening in the Fisheries Sector. Bangladesh. 20 pp. http://www.fao.org/docrep/field/ 003/ AC377E/AC377E00.htm\#TOC. Accedida el 13 de octubre de 2013

12. Primary Industries And Resources Sa (PIR). 1999. Recirculation sistems in aquaculture. Aquaculture SA. 10 pp.
13. Ricker W. 1971. Methods for assesment of fish production in freshwater. International Biological Programme. Hand Book No.3. Blackwell Scientific Publications, Oxford and Edimburg, 348 pp.

14. Timmons M y Ebeling J. 2007. Recirculation aquaculture. $2 d$ edition. Ithaca. Cayuga Aqua Ventures, $975 \mathrm{p}$.

15. Yilmaz E, Sahin A, Duru M y Akyrut I. 2005. The effect of varying dietary energy on growth and feeding behaviour of common carp. Cyprinus carpio. under experimental conditions. Applied Animal Behavior Science, 92: 85-92.

16. Yousef S, Hafedh A, Aftab A y Afaque A. 2003. Performance of plastic biofilter media with different configuration in a water recirculation system for the culture of Nile tilapia (Oreochromis niloticus). Aquaculture Enginnering, 29:139-154.

17. Zar J. 2009. Biostastistical Analisys. 5th edition. New Jersey, Prentice Hall; 2009, 526 p. 